\title{
LA DISPOSICIÓN A APLICAR ESTANDARES EN EMPRESAS PRODUCTORAS DE HORTALIZAS EN EL NOROESTE DE MÉXICO
}

\section{THE WILLINGNESS TO APPLY STANDARDS IN VEGETABLE MANUFACTURING COMPANIES IN NORTHWEST MEXICO}

\author{
Antonio M. Martínez \\ Recibido para publicación: Diciembre 21 de 2013 - Aceptado para publicación: Septiembre 27 de \\ 2014
}

\begin{abstract}
RESUMEN
El proceso de certificación de las empresas productoras de hortalizas en la Región Noroeste de México, se hace más necesario, si se quiere mejorar la competitividad, como respuesta a exigencias mismas del mercado. Este trabajo busca hacer una medición cuantitativa de la Disposición a Certificarse por parte de los productores, lo cual implica asumir costos adicionales pero que luego se convierten en ventajas comparativas en la medida que los productos puedan llegar al mercado. Mediante el uso del método de valoración contingente (VC), técnicas de regresión logística con estimación de parámetros usando máxima verosimilitud, se concluye que las variables aplicación de estándares especialmente de calidad e inocuidad, trabajar bajo agricultura por contrato influyen positivamente en la Disposición a Certificar la producción; mientras que la experiencia como productor de hortalizas, influyen negativamente.
\end{abstract}

Palabras clave: Calidad, inocuidad, contrato, competitividad, mercados

\begin{abstract}
The process of certification of vegetable producers in the northwest region of Mexico, it becomes necessary, If you want to improve competitiveness, in response to market demands them. This paper seeks to make a quantitative measurement of the provision to Certify by farmers, which means assuming additional costs but then become comparative advantages to the extent that the products can reach the market. Using the contingent valuation method (VC), Logistic regression techniques to estimate parameters using maximum likelihood, It is concluded that the application of standard variables especially quality and safety, working under contract farming, positively influence the willingness to certify production; while the experience as a producer of vegetables, influence negatively.
\end{abstract}

Key words: Quality, safety, contract, competitiveness, market

\footnotetext{
${ }^{1}$ Doctor en Ciencias Económicas Investigador PhD. Centro de investigación Turipaná CORPOICA kilómetro 13 vía Montería - Cereté, Córdoba, Colombia. Teléfono 0914227300 Ext 2224 correo electrónico antoniomarti40@ hotmail.com
} 


\section{INTRODUCCIÓN}

La producción agrícola en general tiene como fin generar bienes una vez culmina el proceso de producción primaria que pueden ser alimentos y materias primas que son usados para la producción de otros bienes. Históricamenente, se ha privilegiado la producción física y los rendimientos en cantidad dejando a un segundo plano las calidades de los productos, esto porque no se consideran un tangible vendible en la medida que los mercados no reconocen estos valores al momento de realizar las transacciones y fijar los precios.

El caso de la producción de hortalizas en la región Noroeste de México, no ha sido la excepción, tal vez se debe a que los procesos de certificación son costosos y los productores no ven reflejado este gasto adicional en el precio del mercado del bien o en el precio que paga el consumidor.

En este sentido, se trata de una disposición a incurrir en costos de certificación por parte del productor de hortalizas. El tema de la disposición a invertir en certificación en este caso se aborda bajo el enfoque de Valoración de Contingente (VC) este enfoque se ha utilizado principalmente para la valoración económica de servicios ambientales, pero dado que se trata de expresar un deseo o preferencia revelada, se considera pertinente para poder establecer por una parte si el productor estaría dispuesto a asumir el costo y por otra conocer las razones o variables que explican es decisión.

A manera de justificación, en el caso de la necesidad de que las unidades productivas certifiquen su producción la razón principal consiste en que se debe mejorar la competitividad, como respuesta a exigencias mismas del mercado donde la diferenciación del producto con características de calidad e inocuidad, denominación de origen tienen amplia relación con los aspectos ambientales y en este sentido se trata de hacer una medición cuantitativa de la disposición a certificarse por parte de los productores de hortalizas de la región Noroeste de México.

La región Noroeste de México comprende los estados de Sinaloa, Sonora, Baja California Sur y Baja california Norte con gran dinamismo en la producción de hortalizas con destino a la exportación. De acuerdo con las estadísticas de la Secretaría de Agricultura, Ganadería, Desarrollo Rural, Pesca y Acuicultura SAGARPA, Las hortalizas presentan una participación promedio del $16 \%$ del valor de la producción agrícola. Su tasa de crecimiento promedio es del 6,7\% en el periodo 2000 - 2010 su participación con relación a las exportaciones agrícolas fue del 58\% para el subsector agrícola. La balanza comercial de hortalizas es superavitaria con un valor de 2.392.160 dólares y este superávit ha venido aumentando, en el 2008 registró una tasa de crecimiento del $0,8 \%$ que en valor corresponde a 19.137,28 dólares los cuales son divisas que le entran al país (SAGARPA 2010).

La producción de hortalizas en esta región, es una actividad económica relevante, lo cual se atribuye a factores naturales como el clima, calidad de los suelos y la cercanía al mercado de Estados Unidos de Norteamérica. Igualmente ha creado ventajas competitivas mediante la diferenciación del producto, por su calidad, a través de la aplicación de estándares y el uso de tecnologías modernas, constituyéndose como la 
región líder en la producción y exportación de hortalizas frescas de México (Martínez 2010).

Con relación a la aplicación de grados y estándares en la producción de hortalizas, se observa que la exigencia de normas para los países en desarrollo no necesariamente es un obstáculo aunque en algunos casos pueden ser barreras para las exportaciones. El hecho de que algunos países o industrias estén utilizando normas de alta calidad y seguridad pone de relieve la importancia de considerar los efectos de la inocuidad de los alimentos de origen agrícola superando las limitaciones que se puedan presentar dentro de la cadena (Henson y Hensman 2004).

La certificación es el procedimiento mediante el cual una tercera parte diferente e independiente del productor y el comprador, asegura por escrito que un producto, un proceso o un servicio, cumple los requisitos especificados, convirtiéndose en la actividad más valiosa en las transacciones comerciales nacionales e internacionales (FAO 2010).

De acuerdo con lo anterior, dentro de la producción de hortalizas la aplicación de estándares y regulaciones valorados y avalados por un ente certificador permitiría ayudar a minimizar las fallas del mercado, además se disminuiría la distancia entre comprador y vendedor y se disminuirían los costos de transacción especialmente los relacionados con la información a la vez que si la producción se hace aplicando las normas exigidas por el mercado se asegura su participación y se eliminan las barreras de entrada (Martínez 2010).

La hipótesis que subyace en esta investigación es que los productores de hortalizas son consientes de que si aplican buenas practicas agrícolas y siguen con rigor los protocolos podrán generar productos con diferenciales de precios y ser competitivos y para esto necesariamente deben tener la disposición de incurrir en costos adicionales.

El método deValoración Contingente de acuerdo con Tudela (2009) al aplicar los modelos de lección discreta para la valoración económica de servicios ambientales basado en las preferencias declaradas, englobando los experimentos de elección y el método de valoración contingente en sus diferentes versiones que muestran las disposición a pagar.

A pesar que el método de Valoración Contingente se ha aplicado principalmente a estudios de valoración de servicios ambientales, por tratarse de indagar acerca de las preferencias reveladas de cualquier agente económico frente a la decisión de realizar alguna acción, también puede ser aplicable para explicar la intención o disposición por parte del productor de hortalizas de aplicar estándares que lo conduzcan a la certificación de su producción con el fin de generar diferenciales en su producto final que lo haga mucho mas competitivo.

El método de Valoración Contingente en los casos que se ha aplicado como en el trabajo de Tudela, J. (2008). En el estudio de la estimación de la disponibilidad a pagar de los habitantes de la ciudad de Puno en el Perú por el tratamiento de aguas servidas.

El otro estudio de referencia es el deValdivia et al. (2009) que consistió también en una estimación econométrica por el método de máxima verosimilitud acerca de la disponibilidad a pagar por los consumidores de servicios recreativos 
turísticos. En estos dos casos sin querer decir que son los únicos, han dejado en evidencia que este método constituye una herramienta consistente cuando se trata de indagar sobre preferencias reveladas por parte de los agentes económicos y por tanto ha adquirido gran aceptación cuando se trata de formular política pública.

La característica principal del método de valoración contingente con formato referéndum (MVCR), es que se deja al individuo solamente con el problema de decidir si está dispuesto a pagar o no una suma determinada por acceder a los beneficios del programa de conservación que se ofrece. En este evento, todas las posibles posturas o propuestas del encuestador se distribuyen aleatoriamente entre los encuestados; esto es, se determinan previamente las cantidades a ofrecer (Tudela et al. 2009).

La aplicación del método consiste en dejar en libertad al encuestado de manifestar si está dispuesto a pagar o no una suma determinada de dinero a cambio de acceder a un beneficio que deriva del pago. En este caso si estaría dispuesto a invertir unas unidades monetarias adicionales a cambio de conseguir la certificación de sus productos, las hortalizas es decir deciden cambiar su nivel de utilidad cuando se deciden en invertir en la aplicación de protocolos de buenas prácticas agrícola y por eso se denota con la letra u como se muestra en la ecuación.

$$
\mathrm{u}_{i j}=\mathrm{u}_{i}\left(\mathrm{y}_{i}, \mathrm{z}_{j}, \varepsilon_{i j}\right)
$$

Donde:

$U_{\mathrm{ij}}=$ Utilidad derivada de incurrir en costos de certificación. $\mathrm{i}=1$ es cuando se ha implementado.

$\mathrm{i}=0$ es el estado sin cambios o status quo o sin cambios en el costo.

Determinantes de la utilidad $y_{i}$, el costo de producción del j-ésimo encuestado.

$\mathrm{Z}_{\mathrm{j}}$, un vector de atributos y características de la producción y

$E_{i j}$, un componente de preferencias que revela el encuestado en forma individual pero no observadas por el investigador.

La probabilidad de una respuesta positiva es la probabilidad de que el encuestado piense que estará en condiciones de incurrir en un costo adicional dado a la utilidad que deriva de usar una unidad monetaria adicional en implementar protocolos de buenas prácticas agrícolas, por tanto $\mathrm{u}_{1}>\mathrm{u}_{0}$. Para el encuestado j, esta probabilidad es:

$\operatorname{Pr}(s i j)=\operatorname{Pr}\left(u_{1}\left(y_{j}-t_{j}, z_{j}, \varepsilon_{j}\right)>u_{0}\left(y_{j}, z_{j}, \varepsilon_{0 j}\right)\right)$

En estas circunstancias, la probabilidad de que responda afirmativamente sería igual a que quiere decir el número total de encuestas menos los que respondieron negativamente a la pregunta de disposición a incurrir en costos adicionales. En este sentido la función acumulativa de probabilidad de que el consumidor conteste afirmativamente es de tipo logística.

Este trabajo tiene como objetivo entender los diferentes factores que inciden en la decisión por parte del productor de incurrir en costos de certificación con el fin de aumentar la competitividad de la producción de hortalizas, aplicados a la región noroeste de México, en este sentido a través de una encuesta el productor manifiesta su preferencia por tener su empresa certificada. 


\section{MATERIALES Y MÉTODOS}

La información de este artículo se obtuvo en el trabajo de campo del proyecto "Estándares e Instituciones Competitividad Limitada financiado con recursos del ejecutado por la Universidad Autónoma de Baja California.

El marco geográfico para este estudio fue la Región noroeste de México que comprende los estados de: Baja California, Baja California Sur, Sinaloa y Sonora. La información utilizada fue producto de la aplicación de una encuesta a 58 agricultores de la Región Noroeste de México donde además de los datos de producción se indagó sobre la disposición a certificar su unidad productiva.

El trabajo de campo constituyó la fuente de información directa e incluye desde la definición de la muestra, número de encuestas a realizar y diseño del cuestionario, aplicación del cuestionario y captura de información complementada con entrevistas a agricultores y gerentes de empresas agrícolas.

Para la definición del tamaño de la muestra se aplicó la técnica de muestreo estratificado con afijación proporcional. En este caso se tomó como variable de muestreo la región, por tanto los estratos son los cuatro estados: Baja California, Baja California Sur, Sonora y Sinaloa y de aquí se calculó el tamaño de la muestra, distribuidas proporcionalmente con un intervalo de confianza de $90 \%$ para un valor de 1,65 y un margen de error del $10 \%$ que da como resultado el número total de encuestas a realizar. La cual dio como resultado una muestra de 58 encuestas aplicadas a igual número de empresas agrícolas, distribuidas así: para Baja California 26 encuestas, para Baja California sur 14 encuestas, para Sinaloa 13 encuestas y 5 para el estado de Sonora.

\section{Diseño del Modelo Econométrico}

Para determinar la disponibilidad de los agricultores de hortalizas a incurrir en costos adicionales en la aplicación de protocolos con el fin de conseguir que su producción sea certificada, se tuvo en cuenta un grupo de variables como edad del agricultor, escolaridad, experiencia en el cultivo, tamaño de la finca, aplicación de buenas prácticas agrícolas, contratos a futuro, las cuales actuaron como variables explicatorias y como variable dependiente la disposición a certificarse.

Es de anotar que de muchas variables que podrían explicar la disposición a pagar solo tres variables presentaron características estadísticas que permitieron una robustez del modelo.

En el caso de las variables cualitativas se establecieron variables binarias y dicotómicas. Las variables de tipo cuantitativo se agruparon por rangos con el fin de hacer menos dispersos los datos y facilitar su manejo.

La información se agrupó en tablas de frecuencia y con la utilización de los programas econométricos Eviews y Stata se estimaron regresiones LOGIT, usando el método de máxima verosimilitud.

\footnotetext{
${ }^{2}$ Valores del Pseudo- $R^{2}$ entre 0,2 y 0,4 se consideran indicativos de un ajuste del modelo relativamente alto (Domencich y McFadden1975). Este valor no tiene la misma interpretación del $\mathrm{R}^{2}$ en las estimaciones por el método de mínimos cuadrados por tratarse en este caso de variables cualitativas y el método de máxima verosimilitud
} 
Los resultados sobre la probabilidad de que las empresas hortícolas se certifiquen se obtuvieron a partir de un modelo logit también conocido como normit, que utilizó McFadden (1973). De acuerdo con Maddala (1996), los modelos de elección binaria asumen que los individuos se enfrentan con una elección entre dos alternativas y la elección depende de características identificables. Maddala (1996); Pindyck y Rubinfeld (2001), argumentan que dichos modelos se refieren a decisiones que involucran "deseo" y "capacidad." Consecuentemente, un modelo como el expresado contendrá variables explicativas de ambos elementos o atributos (Martínez 2010).

La forma inicial de plantear el modelo dicotómico será:

$$
y_{i}=\left\{\begin{array}{lll}
1 & \text { si } & U_{i}^{*}>0 \\
0 & \text { si } & U_{i}^{*} \leq 0
\end{array}\right.
$$

Se trata de establecer la posibilidad de la ocurrencia del hecho donde 1 es si ocurre y 0 si no ocurre. En este caso se supone que la función de distribución es una curva logística por tanto se utiliza el modelo logit donde se relacionan la variable endógena $Y_{i}$ con las variables explicativas $X_{i}$ a través de una función de distribución (Gujarati 2006).

El modelo de probabilidad quedaría definido de la siguiente manera:

$\operatorname{Pr}(s i j)=\operatorname{Pr}\left(u_{1}\left(y_{j}-t_{j}, z_{j}, \varepsilon_{j}\right)>u_{0}\left(y_{j}, z_{j}, \varepsilon_{0 j}\right)\right)$

El Modelo general planteado en este caso fue:

$$
P S 1=\frac{P i}{1-P i} \beta_{0}+\beta_{1} X_{1}+\beta_{2} X_{2}+\beta_{3} X_{3}+\ldots \ldots . \beta_{n} X_{n}+e(2)
$$

Dónde:

Ps1 = Disponibilidad de asumir costos de certificación
$X_{1}, X_{2} X_{3} \ldots \ldots \ldots . . . X_{n}=$ Aplicación de estándares, contrato, experiencia.

El resultado de este ejercicio es determinar las variables que inciden para que el productor se certifique o las razones que explicarían la negativa o la probabilidad de certificar su producción y cuales hacen una contribución mayor al proceso de adopción de estándares por parte de las empresas productoras de hortalizas.

Construcción del Modelo Logit: Con la información obtenida en el trabajo de campo se construyó el modelo Logit, por considerar que los modelos de regresión cualitativa agrupados o de proporciones se consideran los más apropiados para determinar el deseo o actitud del productor frente a la ocurrencia de un hecho en particular, en este caso el deseo o la aspiración del agricultor de que su producción esté certificada.

La presentación de las variables que componen el modelo se puede apreciar en la tabla 1.

La ecuación de regresión para explicar la probabilidad de estar certificadas a través las variables explicativas se muestra así:

$$
P C e r t=\frac{P i}{1-P i}\left(-\left(\beta_{0}\right)+\beta_{1}\right) * \text { ASTAND }+\beta_{2}{ }^{*} \text { CONTRATO }+\beta_{3}^{*}(\text { EXPR })+e
$$

Donde:

Pcert= Disposición a certificar la producción de hortalizas.

$\beta=$ Valor de los parámetros producto de la estimación del modelo Logit

Astand $=$ Aplica estándares en hortalizas.

Contrato $=$ Tiene agricultura por contrato.

Exper $=$ Experiencia como productor

$\mathrm{e}=$ Termino de perturbación. 
Tabla 1. Variables que conforman el modelo para determinar la disponibilidad a invertir en procesos de certificación de la producción de hortalizas en la región Noroeste de México

Variable Dependiente

$$
\begin{array}{cc}
\text { Cert }=1 \quad \text { Si Disponibilidad a Certificarse. } \\
\text { Cero (0) de otra forma. }
\end{array}
$$

\section{Variables Independientes}

$$
\begin{gathered}
\text { Astand }=1 \\
\text { Contrato }=1 \\
\text { Expert }=1
\end{gathered}
$$

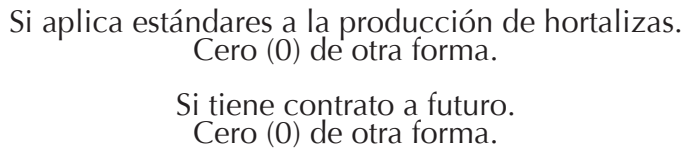

Si tiene experiencia en el cultivo de hortalizas. Cero (0) de otra forma.

Fuente: Elaboración propia 2014

Es de anotar que habrá tantos parámetros como variables independientes haya.

De acuerdo con esta ecuación la probabilidad de explicar la disposición de los agricultores a certificarse puede ser explicada por la aplicación de estándares a la producción especialmente los de calidad e inocuidad, la celebración de contratos a futuro y la experiencia que tenga el productor en cultivar hortalizas.

En la determinación de los factores que explican la probabilidad de certificar la producción de hortalizas por parte de las empresas productoras partió de los siguientes supuestos:
a) Existe una relación directa entre certificarse y aplicar estándares

b) Obtener una certificación implica costos adicionales y no todos podrán están dispuestos a invertir.

c) Tener contratos a futuro implica cumplir con producción de mejor calidad.

\section{RESULTADOS Y DISCUSIÓN}

Una vez definidas las variables y formulado el modelo matemático se procedió a la estimación de los parámetros haciendo uso del método de máxima verosimilitud. Los cuales se presentan a continuación:

La ecuación producto de la estimación de parámetros se explica a continuación (Tabla 2)

$\operatorname{Pr} C e r t=\frac{P i}{1-P i}+\beta_{1}^{*}{ }^{2,805685}+\beta_{2} * 2,508084+\beta_{3} *(-9481277)+e(4)$

El valor del parámetro 2,805685 que corresponde a la variable ASTAND significa que cuando las empresas aplican estándares a su producción, es más factible que estén dispuestos a invertir en el proceso de certificación.

El de la variable CONTRATO, significa que si la empresa realiza agricultura por contrato o celebra contratos con el comprador, la posibilidad de invertir en certificarse será mayor. 
En otras palabras la celebración de contratos que obligan su cumplimiento hace que se interese por tener una producción certificada.

El valor del parámetro de 2,508084 con signo positivo indica que cada vez que una empresa firma un contrato, es posible que las posibilidades de invertir en certificación sea mayor.

La variable EXPERT significa que los productores dado a su experiencia consideran que no seria muy llamativo certificarse y de ahí que este parámetro tiene signo negativo, a mas años de experiencia los productores son mas reacios a hacer cambios a su producción y prefieren seguir produciendo como lo han venido haciendo, por eso el coeficiente tiene signo negativo con un valor de -9481277 .

Lo que significa que la posibilidad de adoptar invertir en procesos de certificación disminuye entre más experiencia tenga el productor.
La información de la tabla 2 muestra los resultados de regresión del modelo logístico dicotómico que representa la disponibilidad a invertir en procesos de certificación por parte de los agricultores de hortalizas de la adopción Región Noroeste de México. La R² de McFadden es de $0,3533^{2}$, indica que el modelo es adecuado para determinar la probabilidad de que el agricultor tenga la disposición a certificar la producción de hortalizas.

Los valores del Z estadístico superan los valores de $z$ de la distribución de probabilidad correspondiente, considerando sus respectivos grados de libertad, por tanto se rechaza la hipótesis nula $\mathrm{HO}$ : $\beta=0$, en este sentido se rechaza la hipótesis nula conjunta de que todos los coeficientes de regresión son cero, al 90 por ciento de confianza.

Tabla 2. Resultados de la estimación del modelo de disponibilidad a invertir en certificación por parte de las empresas hortícolas del Noroeste de México 2014.

\begin{tabular}{|c|c|c|c|}
\hline $\begin{array}{c}\text { Variables Independientes } \\
\text { Dependiente }\end{array}$ & COEFICIENTES & Z-STATIST & PROB \\
\hline ASTAND & 2,805625 & 2,75 & 0,006 \\
\hline CONTRATO & 2,508084 & 2,92 & 0,003 \\
\hline EXPERT & $-0,9481277$ & 1,58 & 0,115 \\
\hline McFadden $\mathbf{R}^{2}$ & 0,3533 & & \\
\hline
\end{tabular}

Fuente: Elaboración propia con datos de la encuesta Universidad Autónoma de Baja California UABC y financiada por el Consejo Nacional de Ciencia y Tecnología de México CONACYT 2014.

\footnotetext{
3 Los efectos marginales significan las contribuciones que hace cada una de las variables independientes en la explicación de la variable dependiente, se expresan en porcentaje de probabilidad que las variables explicatorias contribuyan individualmente. Para el caso que nos ocupa, se trata de estimar en que porcentaje la aplicación de estándares, los contratos y el tiempo que lleva como productor de hortalizas contribuyen en la explicación en la disposición a invertir dineros en procesos de certificación por parte de las empresas productoras de hortalizas de la región Noroeste de México
} 
Cabe anotar que en todos los casos fueron significativos a menos del $10 \%$ y por esta razón se acepta la hipótesis de que los $\beta$ son diferentes del cero.

Las variables incluidas en el modelo resultaron significativas al 90 por ciento, a excepción de la variable experiencia que no resulto significativa con un margen de error del $11 \%$, por tratarse de investigación en campo no controlada para las pruebas de significancia se tomó un margen de error del $10 \%$ por considerar que los datos se obtuvieron de campo a través de encuestas y no es de experimentos controlados por eso el margen del error permitido es del 10\%.

Cuando se analizaron en forma conjunta (Tabla 2), esto demuestra que un cambio en algunas de las variables independientes ocasionan cambios en la probabilidad de que las empresas esté dispuestas a incurrir en costos de certificación.

Cuando se calcularon los efectos marginales ${ }^{3} \mathrm{o}$ las contribuciones individuales, las tres variables resultaron significativas. Se observa que aplicar estándares en la producción es el primer paso para la certificación y fueron los que mostraron una mayor contribución en todo el modelo estimado con un valor de 0,51 ; indica que un aumento en una empresa de las encuestadas que aplique estándares hace aumentar en 51 por ciento la probabilidad de que las empresas estén dispuestas a invertir en procesos de certificación. Lo anterior se explica por que como se anotó anteriormente la aplicación de estándares y la cultura de aplicar los estándares es el primer paso para la certificación.

La agricultura por contrato no solo crea un ambiente de estabilidad y confianza al productor, sino que además obliga de cierta manera al agricultor que cumpla con las normas y estándares que exige el mercado. La contribución en la disposición de invertir en los procesos de certificación es de 0,33; muestra que una empresa de las encuestadas que celebre contratos aumentaría la probabilidad de certificarse en un 33 por ciento.

Por último los años que lleva como productor de hortalízas hace una contribución individual con un valor de $-0,11$; en este caso entre más años tenga el productor menos probabilidad de que invierta en procesos de certificación.

A través del análisis realizado a las empresas hortícolas encuestadas en la Región Noroeste de México se demuestra que estas incrementan la probabilidad de certificarse y la disposición a incurrir en costos de certificación en la medida que apliquen estándares y celebren agricultura por contrato.

Considerando la variables que presentaron mejores características estadística en la explicación de la disposición a certificarse las respuestas obedecen a las revelaciones de cada uno de los agricultores encuestados, es muy común encontrar en más de un 80 por ciento de la muestra a agricultores que trabajan bajo el concepto de Buenas Prácticas Agrícolas, además por tratarse de un producto cuyo destino es la exportación, la presencia de contratos de producción y comercialización es un factor que motiva a buscar una certificación como paso previo a obtener una marca.

\section{CONCLUSIONES}

A pesar que la valoración contingente es un método utilizado para determinar valores que el 
mecanismo de los precios no alcanza a detectar y se base en preferencias reveladas por parte del consumidor, en el caso de la disposición a invertir en procesos de certificación en la producción de hortalizas permitió determinar los factores que hacen posible la disposición a invertir sin detrimento de la técnica ni de la teoría sobre la cual descasa este método de medición.

La aplicación del método de Valoración contingente en el caso de la producción y comercialización de hortalizas en la región noroeste de México permitió entender cómo la aplicación de estándares y la celebración de contratos a futuro permiten entender la importancia de la certificación de la producción en la búsqueda de un mejor posicionamiento en el mercado que lo haga mas competitivo.

A través del análisis se demuestra que las empresas productoras de hortalizas de la Región Noroeste de México incrementan la probabilidad de la Disponibilidad de Costear los procesos de certificación en la medida que se entienda que el inicio del proceso es la aplicación de estándares y que contar con una certificación le garantiza un mayor precio en el mercado que le permite recuperar la inversión.

\section{REFERENCIAS}

Domencich and McFadden 1975. illustrates the conceptual flexibility and computational drawbacks of this approach. Journal of Economics.

FAO. 2010. Organización de las naciones unidas para la agricultura y la alimentación) en Httpp//www.fao.org [noviembre de
2010].

Gujarati, D. 2006. Econometría. 4 ed., México, MacGraw Hill.

Henson, S. and Hensman, M. 1998. Food Safety Regulation and the Firm. Understanding the Compliancen Process. Food Policy 23(1): 9-23.

Herrador, D. y Dimas, L. 2001. Valoración económica del agua para el área metropolitana de San Salvador. Programa Salvadoreño de investigación sobre Desarrollo y Medio Ambiente (PRISMA). El salvador. 65p

Maddala, G. 1996. Introducción a la econometría. 2. ed. México, PrenticeHall.

Martinez, A. 2010. Instituciones y Estándares: su rol en el sector hortícola del noroeste de México. Tesis de doctorado Universidad Autónoma de Baja California. 173p.

McFadden, D. 1973. Conditional logit Analysis of Qualitative Choice Behavior. En P. Zarembka, Frontiers in Econometrics. Nueva York, Academic Press.

McFadden, D. 1975. The revealed preferencies of government bureavecracy Theory Bell Journal of Economics 6: 401-416.

Pindyck, R. y Rubinfel D. 2001. Econometría: Modelos y pronósticos, 4 ed., México, McGraw Hill. Econometría: Modelos y pronósticos, 4 ed., México, McGraw Hill. Polése. 
Secretaría de agricultura, ganadería, desarrollo rural, pesca y alimentación SAGARPA. 2010. Estadísticas de Producción Agropecuaria disponibles en www. sagarpa.gob.mx fecha de consulta octubre 13 de 2010.

\section{Tudela , J., Martínez, M., Valdivia, R., Portillo V.} y Romo, J. (2009). Modelos de elección discreta en la valoración económica de áreas naturales protegidas. Rev. Mex. Ec. Agríc. Rec. Nat. 2: 7-29.
Valdivia A., Cuevas, C., Sandoval V. y Romo, J. (2009). Estimación econométrica de la disponibilidad a pagar por los consumidores de servicios recreativos turísticos. Terra Latinoamericana 27: 227235.

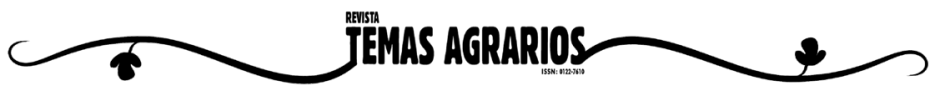

